\title{
Elaboración de productos panificados a partir de premezcla de harinas aptas para celíacos enriquecidas con hierro y vitaminas del complejo B y su aceptabilidad
}

Pamela Violeta Cubilla-Giménez, María Belén Monsalvo-Benítez, José Acosta-Escobar, Laura Elizabeth González-Céspedes, Macarena Morínigo-Martínez*

Departamento de Nutrición. Dirección de Investigación. Facultad de Ciencias Químicas. Universidad Nacional de Asunción, Paraguay

Cómo referenciar este artículo/ How to reference this article:
Cubilla-Giménez PV, Monsalvo-Benítez MB, Acosta-Escobar J, González-Céspedes LE, Morínigo-Martínez M. Elaboración de productos panificados a partir de premezcla de harinas aptas para celiacos enriquecidas con hierro y vitaminas del complejo B y su aceptabilidad. Mem. Inst. Investig. Cienc. Salud. 2018; 16(1): 64-70

\section{R E S U M E N}

La Ley 20.830/98 reglamenta el enriquecimiento de la harina, sin embargo, no contempla a la premezcla de harinas aptas para celíacos. Aproximadamente $50 \%$ de los celíacos presenta anemia, por lo es necesario elaborar productos cuyas características nutricionales, sensoriales y funcionales sean óptimas. El objetivo del trabajo fue enriquecer con hierro y vitaminas del complejo B (mix) la premezcla de harinas apta para celíacos y evaluar la aceptabilidad de dos panificados de alta demanda poblacional elaborados a partir de la premezcla. En cocinas especializadas para celíacos se elaboraron panes y palitos, cada uno en dos versiones (con mix y sin mix), se presentaron en envases individuales y etiquetados forma numérica para la degustación. Se evaluó la aceptabilidad de los panificados utilizando una escala hedónica de 9 puntos a través de un cuestionario autoadministrado. Se utilizó la prueba t de Student para la comparación de promedios obtenidos. Participaron 105 celíacos ( $81 \%$ mujeres), el promedio de edad fue 34,5 $\pm 10,9$ años Según auto-reporte el $60 \%$ cursó con anemia. La puntuación global promedio de aceptabilidad fue: pan sin mix $7,42 \pm 1,33$ puntos; pan con mix $8,00 \pm 1,06$ puntos; palito sin mix $7,91 \pm 1,05$ puntos y palito con mix 7,86 1,17 puntos. Se realizó el análisis de la premezcla apta para celíacos y se constató que la misma cumple con el aporte de micronutrientes (hierro, B1, B2, B3 y ácido fólico). En este estudio, los panes con mix tuvieron mayor aceptabilidad que aquellos sin mix. En el caso de los palitos no se observaron diferencias en la aceptabilidad entre productos (con mix vs sin mix).

Palabras clave: hierro, vitaminas del complejo B, premezcla de harinas, celiaquía.

\section{Preparation of bakery products from premix of flours suitable for celiac people and enriched with iron and vitamin B complex and their acceptability}

\author{
A B S T R A C T \\ Law $20,830 / 98$ regulates the enrichment of flour, however, it does not contemplate the \\ premix of flours suitable for celiac people. Approximately $50 \%$ of celiac patients have \\ anemia, so it is necessary to elaborate products whose nutritional, sensory and functional \\ characteristics are optimal. The objective of the work was to enrich with iron and vitamin B \\ complex (mix) the pre-mix of flours suitable for celiac people and to evaluate the \\ acceptability of two high-demand bakery products made from the pre-mix. In specialized \\ kitchens for celiac people, breads and sticks were made, each in two versions (with mix and


without mix), presented in individual packages and labeled in numerical form for tasting. The acceptability of the bakery products was evaluated using a hedonic scale of 9 points through a self-administered questionnaire. The Student's t test was used to compare the averages obtained. One hundred and five celiac patients participated ( $81 \%$ women) and the mean age was $34.5 \pm 10.9$ years. According to self-report, $60 \%$ had anemia. The average global acceptability score was: bread without mix $7.42 \pm 1.33$ points, bread with mix $8.00 \pm 1.06$ points, stick without mix $7.91 \pm 1.05$ points and stick with mix $7.86 \pm 1.17$ points. The analysis of the pre-mix suitable for celiac people was carried out and it was found that it meets the contribution of micronutrients (iron, B1, B2, B3 and folic acid). In this study, breads with mix had greater acceptability than those without mix. In the case of the sticks, no differences were observed in the acceptability between products (with mix vs. without mix).

Keywords: iron, B vitamin complex, flour premix, celiac.

\section{INTRODUCCIÓN}

La enfermedad celíaca (EC) es una enteropatía provocada por una reacción autoinmune a proteínas del gluten (gliadinas y gluteninas), hordeínas y secalinas, en individuos genéticamente susceptibles. Estas proteínas de reserva (ricas en glutamina) son necesarias para la germinación de los granos del trigo, centeno y cebada ${ }^{(1)}$.

La anemia ferropénica es un tipo de anemia atribuible a una mayor pérdida de hierro o bien a la alteración de la absorción del mismo cuyas consecuencias impactan negativamente sobre la salud. Las estimaciones realizadas a nivel mundial indican que un tercio de las personas no alcanzará su potencial físico e intelectual total debido a la carencia de micronutrientes. Con base en estas predicciones un gran número de países latinoamericanos han establecido el enriquecimiento obligatorio de las harinas de trigo, teniendo en cuenta que los productos derivados de ella son de consumo masivo por la población ${ }^{(2)}$.

La deficiencia de hierro en la EC se produce principalmente como consecuencia de la absorción anormal de este micronutriente, sin embargo, también podría deberse a la pérdida de sangre oculta en el tracto gastrointestinal. La anemia ferropénica es un síntoma que generalmente se observa en la EC $(\sim 50 \%)^{(3)}$. Si bien una minoría de los pacientes con EC presentan síntomas de mala absorción clásica, el mayor número de ellos se ve afectado por las formas subclínicas o silenciosas ${ }^{(4)}$.

En abril de 1998 por medio del decreto 20830/98 Paraguay declaró obligatorio el enriquecimiento de las harinas de trigo como estrategia nacional de salud pública para la prevención y control de deficiencias de hierro en la población general. Sin embargo, al ser el trigo un alimento con alto contenido en gluten y por ende de consumo restringido en los pacientes con EC, las premezclas preparadas a base de harinas de arroz, almidón de maíz, almidón de mandioca, entre otras no quedan amparadas por este decreto ${ }^{(5)}$.

Teniendo en cuenta la elevada prevalencia de anemia en la población celíaca, se considera importante la fortificación de la premezcla de harinas aptas para celíacos de manera a mitigar esta situación. Al fortificar un producto de consumo masivo y elevada aceptación como los panificados, se pretende brindar al paciente micronutrientes que se ven encarecidos por la propia patología, sin embargo, el gusto de los alimentos fortificados con hierro podría verse afectado, debido a que el mismo es un quelante con sabor metálico.

Por todo lo mencionado anteriormente, el objetivo de esta investigación consistió en enriquecer con hierro y vitaminas del complejo B la premezcla de harinas apta para celíacos y evaluar la aceptabilidad de panificados (panes y palitos) elaborados a partir de esta premezcla, de forma a contribuir de manera activa en la creación de opciones de alimentos que cumplan con lo establecido en el marco legal y se enfoquen a satisfacer las demandas de un segmento importante de la población.

\section{MATERIALES Y MÉTODOS}

Se diseñó un estudio analítico transversal, en el que se incluyó a personas de ambos sexos, de entre 19 a 59 años, con EC, miembros de la Fundación Paraguaya del Celiaco (Fupacel) y que estuvieran dispuestos a participar de la evaluación. Se excluyó a todos aquellos que tuvieron diagnóstico reciente de la enfermedad (hasta un año), que decidieron no participar del estudio, a aquellos que presentaban alergia a algunos de los ingredientes utilizados para la elaboración de los panificados, se excluyó también a los que presentaban 
problemas de percepción de olor y/o sabor y a los que residían fuera del Departamento Central.

No habiéndose encontrado estudios que evalúen la aceptabilidad de productos en pacientes con EC y teniendo en cuenta que el montaje típico para la aplicación de escala hedónica es de 80 degustadores, se decidió incluir a todas las personas que cumplan con los criterios de selección utilizando la base de datos propia de la Fundación Paraguaya de Celiacos (Fupacel) y las personas que aceptaron participar de la investigación fueron entrevistadas en sus domicilios.

Para la obtención de los panificados, en un primer paso se elaboró la premezcla de harinas aptas para celíacos. Se utilizó almidón de maíz (40\%), almidón de mandioca (30\%) y harina de arroz (30\%). Por cada kilogramo de premezcla se agregó conteniendo 4,5 mg de Nitrato de monotiamina, $35 \mathrm{mg}$ de niacinamida, 2,5 mg de rivoflavina, $3 \mathrm{mg}$ de ácido fólico y $45 \mathrm{mg}$ de hierro según lo referido en el decreto 20830/98.

Un profesional del área de tecnología de los alimentos verificó el proceso de enriquecimiento y homogenización de la premezcla, en este proceso se empleó una batidora industrial de marca Gpaniz de acero inoxidable con capacidad de $10 \mathrm{~kg}$. Posteriormente se realizó el análisis bioquímico de la premezcla de harina sin gluten, el cual incluyó: análisis químico de hierro, tiamina, niacina, rivoflavina y ácido fólico. La metodología de medición para el hierro fue espectrofotometría UV Visible utilizando el método de orto-fenantrolina y de los demás micronutrientes cromatografía de líquidos de alto rendimiento. Una vez comprobado el cumplimiento del decreto de enriquecimeinto de la harina, se elaboraron los productos panificados a partir de la premezcla con y sin mix. En las reuniones de la Fupacel se procedió a la recolección de datos respecto a la aceptabilidad y adherencia a DLG (Dieta Libre de Gluten).

Se consideró como variable predictora, el tipo de producto: Panificados (panes y palitos) libres de gluten enriquecidos con hierro y vitaminas del complejo B (con mix) y no enriquecidos (sin mix) y como variable resultante la aceptabilidad: medida en puntaje a partir de la escala hedónica. Puntaje en base color, olor, sabor, textura, apariencia, dureza y preferencia global ${ }^{5}$. Además se midieron variables como edad (años), sexo (masculino/femenino), antecedentes de anemia ferropénica medidos por auto reporte respondiendo si padeció o no con anemia y la composición química Hierro (mg), Tiamina (mg); Rivoflavina (mg); Niacina (mg); Acido fólico (mg.) y el cumplimiento de las recomendaciones según la ley 20830/98

Se utilizaron encuestas y análisis sensorial mediante escala hedónica con puntaje del 1 al 9 (1 punto me disgusta muchísimo, 2 puntos me disgusta mucho, 3 puntos me disgusta moderadamente, 4 puntos me disgusta poco, 5 puntos no me disgusta ni me gusta, 6 puntos me gusta poquito, 7 puntos me gusta moderadamente, 8 puntos me gusta mucho y 9 puntos me gusta muchísimo). Esta prueba es una herramienta muy efectiva en el diseño de productos y cada vez se utiliza con mayor frecuencia debido a que son los consumidores quienes, en última instancia, convierten un producto en éxito o fracaso.

Finalmente, los datos fueron digitalizados en el programa informático Microsoft ${ }^{\circ}$ Office Excel 2010 y luego de comprobar la consistencia de la base de datos, se procedió al análisis de los mismos; los datos cuantitativos con distribución normal se resumieron en: promedio y desviación estándar (DE) e intervalo de confianza (IC); los datos cualitativos, se resumieron en frecuencia ( $n$ ) y porcentaje (\%). Para la comparación de datos cuantitativos, se empleó la prueba $t$ de Student en los datos con distribución normal. El valor de $p$ designado fue $p<0,05$. Se respetaron los 3 principios de ética y los productos fueron elaborados en una cocina habilitada por el Instituto Nacional de Alimentación y Nutrición (INAN), teniendo en cuenta las normas de buenas prácticas de manufactura (BPM) y las mayores precauciones a fin de evitar la contaminación cruzada. El protocolo de investigación fue aprobado por el Comité de Ética de la Facultad de Ciencias Químicas de la UNA.

\section{RESULTADOS}

La muestra estuvo conformada por 105 pacientes adultos con diagnóstico previo de EC. Entre las características demográficas se destaca que la mayoría de los pacientes ( $81 \%$, $\mathrm{n}=85$ ) eran del sexo femenino. El promedio de edad de la muestra fue de $34,5 \pm 10,9$ años (IC95\% 32,4-36,6). Al realizar la clasificación por rango de edad, el 36,1\% tenía entre 30 y 39 años (Tabla 1). 
Tabla 1. Características demográficas de los pacientes con enfermedad celíaca

\begin{tabular}{lll}
\hline Datos demográficos & & n (\%) \\
\hline \multirow{2}{*}{ Sexo } & Femenino & $85(81,0)$ \\
& Masculino & $20(19,0)$ \\
\hline \multirow{2}{*}{ Rango de edad } & 19 a 24 años & $35(33,3)$ \\
& 30 a 39 años & $38(36,1)$ \\
& 40 a 49 años & $20(19,0)$ \\
& 51 a 59 años & $12(12,6)$ \\
\hline
\end{tabular}

Alrededor de 6 de cada 10 pacientes encuestados referenció haber padecido anemia en el curso de su condición celíaca, recibiendo en mayor proporción suplementos con hierro $(73 \%)$ como tratamiento al mismo.

Se compararon los promedios de puntajes de aceptabilidad de las muestras de panes sin mix y con mix (hierro, rivoflavina, niacina, tiamina y ácido fólico) respectivamente, se encontró que para todas las características organolépticas el puntaje de aceptabilidad fue mayor en el pan con mix versus sin mix. Las diferencias fueron significativas en todas las características a excepción del olor. Teniendo en cuenta el puntaje global de la escala hedónica, se encontró que el pan sin mix obtuvo un puntaje correspondiente a "me gusta moderadamente", mientras que el pan con mix obtuvo un puntaje promedio de "Me gusta mucho".

Al analizar las diferencias entre los promedios de puntajes de las muestras de palitos sin mix y con mix respectivamente no se encontraron diferencias significativas en ninguna característica organoléptica evaluada, ambas muestras (con y sin mix) fueron organolépticamente aceptables, con puntajes similares (Tabla 2).

Tabla 2. Comparación de promedios de características organolépticas en muestras de panes y palitos con y sin el agregado de mix de micronutrientes.

\begin{tabular}{|c|c|c|c|c|c|}
\hline \multicolumn{2}{|c|}{ Características organolépticas } & \multirow{2}{*}{$\begin{array}{l}\begin{array}{l}\text { Sin Mix } \\
\text { Promedio } \pm \text { DE }\end{array} \\
7,46 \pm 1,56\end{array}$} & \multirow{2}{*}{$\begin{array}{l}\begin{array}{l}\text { Con Mix } \\
\text { Promedio }\end{array} \\
8,02 \pm 1,17\end{array}$} & \multirow{2}{*}{$\begin{array}{l}\text { Diferencia } \\
0,562\end{array}$} & \multirow{2}{*}{$\frac{p^{*}}{0,004}$} \\
\hline \multirow{7}{*}{ Panes } & Color & & & & \\
\hline & Olor & $7,34 \pm 1,71$ & $7,74 \pm 1,50$ & 0,4 & 0,073 \\
\hline & Sabor & $7,28 \pm 1,76$ & $7,83 \pm 1,61$ & 0,552 & 0,019 \\
\hline & Textura & $7,41 \pm 1,52$ & $8,02 \pm 1,21$ & 0,61 & 0,002 \\
\hline & Dureza & $7,35 \pm 1,45$ & $8,19 \pm 0,97$ & 0,838 & 0,001 \\
\hline & Apariencia & $7,70 \pm 1,24$ & $8,22 \pm 1,05$ & 0,514 & 0,001 \\
\hline & Puntaje global & $7,42 \pm 1,33$ & $8,00 \pm 1,06$ & 0,579 & 0,001 \\
\hline \multirow{7}{*}{ Palitos } & Color & $7,87 \pm 1,28$ & $7,88 \pm 1,36$ & 0,01 & 0,958 \\
\hline & Olor & $8,05 \pm 1,16$ & $7,94 \pm 1,33$ & 0,105 & 0,544 \\
\hline & Sabor & $7,87 \pm 1,26$ & $7,88 \pm 1,34$ & 0,01 & 0,958 \\
\hline & Textura & $7,76 \pm 1,22$ & $7,79 \pm 1,45$ & 0,029 & 0,878 \\
\hline & Dureza & $7,72 \pm 1,37$ & $7,59 \pm 1,55$ & 0,133 & 0,509 \\
\hline & Apariencia & $8,20 \pm 0,89$ & $8,10 \pm 1,10$ & 0,105 & 0,449 \\
\hline & Puntaje global & $7,91 \pm 1,05$ & $7,86 \pm 1,17$ & 0,049 & 0,749 \\
\hline
\end{tabular}

1 Sin mix: premezcla de harinas sin el agregado micronutrientes.

2 Con mix: premezcla de harinas con el agregado de hierro, rivoflavina, niacina, tiamina y ácido fólico.

* Prueba t de Student para muestras relacionadas.

En el análisis bioquímico de la premezcla apta para celíacos, enriquecida con hierro, B1, B2, B3 y ácido fólico, se observó que la cantidad de micronutrientes presentes en la premezcla se adecuaba a los valores establecidos en la Ley 20830/98 que el enriquecimiento de harinas de trigo e incluso se observó un alto contenido de tiamina, hierro y ácido fólico respectivamente, comparado con los valores de referencia (Tabla 3). 
Tabla 3. Resultado análisis bioquímico de las cantidades de micronutrientes presentes en la premezcla apta para pacientes con enfermedad celíaca

\begin{tabular}{llll}
\hline & Muestra & Ley 20830/98 & \% de adecuación \\
\hline Hierro (mg) & 59,3 & 45 & 132 \\
Ácido fólico (mg) & 3,25 & 3 & 108 \\
Tiamina (mg) & 31,1 & 4,5 & 691 \\
Rivoflavina (mg) & 2,59 & 2,5 & 104 \\
Niacina (mg) & 35,6 & 35 & 102 \\
\hline$*$ Determinación nutricional realizada en el Laboratorio de Bioquímica & \\
Extensión Universitaria de la FCA-UNA. Metodología AOAC-2000 de la Dirección de \\
** Decreto 20830/98 para la fortificación de harinas de trigo \\
*** Mix vitamínico adquirido de proveedor nacional autorizado por el INAN, en la proporción de 1g para 5kg \\
de harina.
\end{tabular}

\section{DISCUSIÓN}

En general, la EC se da con mayor frecuencia en las mujeres que en los hombres, en una razón $2: 1$, en el presente estudio se encontró que 8 de cada 10 participantes eran del sexo femenino en donde la razón mujer-hombre se vio superada ampliamente, lo cual pudo deberse a que la toma de datos se realizó según el registro de participantes proveído por la Fupacel, donde las mujeres asisten con mayor regularidad. El promedio de edad en la que se encontraban los participantes fue de 34 años.

Los resultados reflejaron que más de la mitad de las personas que poseen enfermedad celiaca presentaron anemia ya sea antes o después de su diagnóstico. A nivel mundial se registra una prevalencia de entre $80-90 \%$ de anemia ferropénica en pacientes celíacos y de entre $12 \%$ a $41 \%$ para anemias por déficit de ácido fólico y vitamina $B 12$, el número de pacientes celiacos con algún grado de anemia es del $49 \%$. Por otro lado Karnam encontró $2,8 \%$ de pacientes celiacos no sospechados en un estudio prospectivo en adultos con anemia ferropénica en quienes se realizó endoscopía con biopsias duodenales y anticuerpos antiendomicio Estos datos sugieren que la anemia por deficiencia de hierro es una manifestación frecuente en adultos. En Paraguay no se registran datos de prevalencia de anemia en celiacos, pero a juzgar por los hallazgos del presente trabajo de investigación y concordando con los datos de la bibliografía citada, aproximadamente 6 de cada 10 participantes refirieron haber padecido anemia ${ }^{(6,7)}$.

El tratamiento de la anemia se basa primeramente en la corrección de la malabsorción por medio de la DLG, al mismo tiempo se indican suplementos de hierro ya sea por vía oral o intravenosa dependiendo de la gravedad ${ }^{(8)}$.

Los países latinoamericanos han implementado diversas estrategias para combatir la anemia. En Paraguay los esfuerzos están dados en términos de medidas higiénicas, desparasitación, consumo de suplementos y el enriquecimiento de alimentos de consumo masivo por la población, como es el caso de las harinas de trigo con hierro y vitaminas del complejo B según el decreto 20830/98. Las premezclas de harinas aptas para celiacos se encuentran exentas de esta normativa debido a que las mismas no contienen trigo. El trigo, al ser fuente de gluten, contiene proteínas que en contacto con la mucosa intestinal genera reacciones autoinmunes lo cual altera la absorción de los nutrientes en los pacientes con EC. Sin embargo, se ha visto que un tiempo después de iniciada la DLG las vellosidades atrofiadas se ven recuperadas. Es decir, los celiacos se verían favorecidos si se enriquecen los productos que consumen diariamente, tal como los panificados sin gluten, pues ésta sería una estrategia viable para revertir la anemia junto con los tratamientos propios de la patología, esperando así reducir al mínimo los efectos secundarios.

Según un estudio realizado por Hurrell $\mathrm{R}$ et $a{ }^{\left({ }^{(9)}\right.}$, las cantidades propuestas de nutrientes para el enriquecimiento de harinas de trigo genera un impacto positivo con los que se ha demostrado una mejora en el estado nutricional referente al hierro en diferentes poblaciones. En cuanto al ácido fólico se han realizado estudios en Estados Unidos ${ }^{(9)}$, Canadá(10) y Chile ${ }^{(11)}$ donde se han registrado descensos del $26 \%$, $42 \%$ y $40 \%$, respectivamente, en la tasa de nacimientos con anomalías congénitas del tubo neural. Se debería incluir en las normativas técnicas y legales a la población celiaca como una estrategia para mejorar su estado nutricional agregando a todo esto que si la anemia no es tratada existen alteraciones de la capacidad de trabajo físico y de la actividad motora espontánea, alteraciones de la inmunidad celular y de la capacidad bactericida de los 
neutrófilos, además de alteraciones funcionales e histológicas del tubo digestivo, falla en la movilización de la vitamina A hepática, mayor riesgo de parto prematuro y de morbilidad perinatal entre otras consecuencias.

La proporción de harinas para la elaboración de premezcla sin TACC es de $45 \%$ para harina de arroz, 30\% para almidón de maíz y $25 \%$ almidón de mandioca ${ }^{(12)}$, por el contrario la harina sin gluten utilizada en este estudio fue de 40\% almidón de maíz, 30\% almidón de mandioca al igual que la harina de $\operatorname{arroz}^{(13)}$ debido a pruebas preliminares realizadas en donde ésta última brindaba un producto menos quebradizo y más parecido al pan con gluten, ya que según Altindag et al. (2014) ${ }^{(15)}$, la harina de arroz tiene menor capacidad de absorción de agua lo cual brinda un producto seco si se excede en su proporción ${ }^{(16,17)}$.

Al realizar el análisis laboratorial de la premezcla de harinas sin gluten enriquecida con micronutrientes, se observó el cumplimento amplio del decreto e incluso una elevada concentración de tiamina y rivoflavina presente en ella, muy por encima de lo referido en el decreto 20830/98, a pesar del aporte aumentado. Según la bibliografía no se reportan nivel de consumo considerados tóxicos debido a que al ser vitaminas hidrosolubles se eliminan del cuerpo de forma natural en caso de que exista exceso. Por otro lado, al realizar la información nutricional de los productos panificados, se pudo observar que con las porciones el aporte de dichas vitaminas no superaba los requerimientos diarios.

Los panes y palitos enriquecidos que fueron utilizados en la investigación como muestra fueron aceptados organolépticamente descartando la posibilidad de que los quelantes del hierro le brinden un sabor amargo a las muestras. Los resultados arrojaron una diferencia significativa para la muestra 3 que corresponde al pan con mix siendo esta la de mayor aceptabilidad, con los mayores puntajes en color, olor, dureza, textura, apariencia y con un promedio global de $8,00 \pm 1,06$ mientras que en el palito no se hallaron diferencias significativas en cuanto al puntaje. Estos resultados podrían deberse a la mayor similitud que se encuentra para los panes sin gluten con aquellos que si lo contienen, no así en los palitos sin gluten ya que estos tienen mayor similitud con las chipas típicas del Paraguay. Como sugerencia a próximos trabajos de investigación, sería interesante que se evaluara con análisis clínicos si la anemia puede ser revertida con el consumo de dicha harina sin gluten fortificada, además analizar el efecto que tiene el hierro como posible potenciador del sabor y realizar alianzas para llevar a cabo más número de mediciones de los alimentos terminados. A pesar de que la muestra es pequeña y de todas las limitaciones mencionadas, toda la información obtenida de la presente investigación brinda una contribución científica para la salud pública, poniendo en evidencia la realidad de un problema de salud tan importante como la enfermedad celiaca, sobre todo en una población vulnerable y que carece de políticas públicas que se enfoquen a la problemática de llevar una dieta libre de gluten que incluya desde la fiscalización de producción de alimentos sin gluten hasta el etiquetado nutricional. Es importante buscar estrategias para revertir de alguna manera las altas cifras de anemia encontradas. Por lo tanto, estos hallazgos constituyen una señal de alerta que se debe tomar en cuenta para la realización de medidas preventivas que deben estar dirigidas a la población celiaca, siempre teniendo en cuenta el respeto y la consideración a la situación socioeconómica y cultural de este grupo de personas.

\section{CONFLICTOS DE INTERES}

Los autores declaran que no existen conflictos de interés.

\section{REFERENCIAS BIBLIOGRAFÍCAS}

1. Herrera $M$, Hermoso $M$, Quera $R$. Enfermedad celíaca y su patogenia. Rev méd Chile. 2009; 137.

2. UNICEF. La falta de vitaminas y de minerales afecta a una tercera parte de la población mundial. Nueva York. UNICEF; 2004.

3. Annibale B, Severi C, Chistolini A, Antonelli G, Lahner E, Marcheggiano A et al. Efficacy of gluten-free diet alone on recovery from iron deficiency anemia in adult celiac patients. The American Journal of Gastroenterology. 2001; 96 (1):132-137.

4. Bastos Oreiro M. Anemia ferropénica: Tratamiento. Revista Española de Enfermedades Digestivas. 2009;101 (1):70

5. Surco Almendras JAlvarado Kirigin J. Estudio estadístico de pruebas sensoriales de harinas compuestas para panificación. Revista Boliviana de Química. 2011; 28 (2): 79-82.

6. OMS, FAO, UNICEF, GAIN, MI, \& FFI. Recomendaciones sobre el enriquecimiento de la harina de trigo y de maíz. Informe de reunión: Declaración de consenso provisional. Ginebra, Organización Mundial de la Salud, 2009 
7. Allen L, de Benoist B, Dary O, Hurrell R [Editores] Guías para la fortificación de alimentos con micronutrientes, Organización Mundial de la Salud y Organización de las Naciones Unidas para la Alimentación y la Agricultura; 2017

8. Rodrigo L, Garrote JA; Vivas S. (2008). «Enfermedad Celíaca». Med Clin (Barc) 131: 264-70

9. Hurrell $R$, Ranum $P$, de Pee $S$, Biebinger $R$, Hulthen $L$, Johnson $Q$. et al. Revised recommendations for the iron fortification of wheat flour and an evaluation of the expected impact of current national wheat flour fortification programs. Comida Nutr Bull. 2010 Mar; 31 (1 Suppl): S7-21.

10. Polanco I. Libro blanco de la enfermedad celíaca. 1st ed. Madrid: ICM; 2008.

11. Donato H. Anemias: detección, tratamiento y profilaxis. En: Boggiano $E$, Bonadeo $M$, Breitman $F$ et al. (eds): Guías para la supervisión de la salud de niños y adolescentes. Buenos Aires: Sociedad Argentina de Pediatría; 2002. págs.257-262.
12. Allen $\mathrm{L}$ et al., eds. Guidelines on food fortification with micronutrients. Geneva, World Health Organization and Food and Agricultural Organization of the United Nations, 2006.

13. Fortification Initiative. Flour Fortification Initiative country database, Flour Fortification Initiative;2008

14. Organización Mundial de la Salud. Recomendaciones sobre el enriquecimiento de la harina de trigo y de maíz. Informe de reunión: Declaración de consenso provisional. Ginebra, Organización Mundial de laSalud,2009

15. Altindag ESiller F. Effects of Flexible Working Method on Employee Performance: An Empirical Study in Turkey. Bus Econ J. 2014;5(03).

16. Torresani M, Somoza M. Lineamientos para el cuidado nutricional. Buenos Aires: Eudeba; 2009.

17. Asociación Celiaca Argentina. Guía sin TACC. Buenos Aires: ACA; 2014. 